\title{
Non-continuous Piecewise Nonlinear Frequency Modulation Pulse with Variable Sub-pulse Duration in a MIMO SAR Radar System
}

\author{
Yujiu Zhao ${ }^{\mathrm{a}}$, Matthew Ritchie ${ }^{\mathrm{b}}$, Xingyu $\mathrm{Lu}^{\mathrm{a}}$, Weimin $\mathrm{Su}^{\mathrm{a}}$ and Hong $\mathrm{Gu}^{\mathrm{a}}$ \\ a Nanjing University of Science and Technology, Department of Electronic and Optical \\ Engineering, Nanjing 210094, Jiangsu, People's Republic of China; ${ }^{\mathrm{b}}$ University College \\ London, Department of Electronic and Electrical Engineering, London, WC1E 7JE, United \\ Kingdom.;
}

\section{ARTICLE HISTORY}

Compiled February 20, 2020

\begin{abstract}
This paper proposes an implementation of non-continuous piecewise nonlinear frequency modulation (N-PNLFM) signals in MIMO radar which are composed of a sequence of N-PNLFM subpulses. The N-PNLFM subpulse can be divided into three segments, the first and third segment are composed of linear frequency modulation (LFM) waveforms. The LFM waveforms in each N-PNLFM subpulse will be orthogonal in one sequence of our new N-PNLFM signal. A non-linear frequency modulation (NLFM) waveform is in the central component of each N-PNLFM subpulse, the bandwidths of each NLFM waveforms are distributed randomly. Each subpulse can be controlled by different variable parameters in both auto-correlation and cross-correlation functions. In order to suppress the high sidelobes in the new N-PNLFM signal and increase the diversity of signals, each subpulse duration is also distributed randomly. Our proposed PNLFM signals are optimized by applying a particle swarm optimization(PSO) algorithm. Comparing with other PNLFM signals, numerous simulations illustrate that our implementation achieves better performance.
\end{abstract}

\section{Introduction}

Multiple-input multiple-output (MIMO) radar has drawn extensive attention because of its high Degrees Of Freedom (DOFs) and excellent performance in applications such as synthetic aperture radar (SAR) and target detection (J. H. Kim 2015; G. Krieger 2014; J. Li 2007). Waveform design has become one of the most essential elements of MIMO radar (G. Cui 2014; S. Sun 2015), therefore MIMO radar systems demand that the waveforms should also have large time-bandwidth product and low peak-to-average power ratio. Recent researches on orthogonal frequency division multiplex (OFDM) waveforms with low correlation sidelobes are shown in (J. H. Kim 2013, 2015; H. Li 2017; J. Wang 2015; W. Wang 2015; Y. Zhao 2018). Meanwhile, other kinds of waveforms for MIMO radar, which are not orthogonal but still have low correlation properties and good ambiguity function (AF) characteristics can be seen in (C. Gao 2016a,b, 2017).

A piecewise nonlinear frequency modulation (PNLFM) waveform in radar system was first proposed in (E. De Witte 2004; H. D. Griffiths 1994) to suppress range side- 
lobes. According to the simulations proposed in (S. Boukeffa 2011), it has been shown that the nonlinear frequency modulation (NLFM) can reduce the auto-correlation sidelobes. Furthermore, the performance of PNLFM waveforms should be considered in relation to their AF (C. Chen 2008). In one conventional waveform design, chirp signals are combined with different subsequence start frequencies to generate MIMO OFDM waveforms (H. Li 2017; W. Wang 2015). These waveforms have large timebandwidth product, low peak average power ratio and low correlation sidelobes. However, the maximum number of MIMO OFDM waveforms is fixed when the grid of time-frequency scheme in the waveforms is set. This kind of waveform can only be applied into limited SAR range swath scenarios due to range ambiguities, or they will suffer a severe performance degradation. Meanwhile, a novel signal for MIMO radar which is composed of a sequence of PNLFM subpluses is proposed in (C. Gao 2017). A key issue with (C. Gao 2017) is that near the middle of each subpulse, the designed waveform has three independent frequencies, this isn't practical in a real radar transmitter. As a part of Gao's proposed waveform, time interval is required between each subpulse to reduce the sidelobes, it leads to the cost of sacrificing transmitting duration.

In this letter, we propose non-continuous PNLFM orthogonal with different subpulse durations (N-PNLFM-O-DSD) signals for MIMO radar, each N-PNLFM-O-DSD waveform has three segments. The LFM waveforms in the first and third segments in each N-PNLFM subpulse are orthogonal in the total time duration of one N-PNLFMO-DSD signal. The NLFM segment proposed in (Y. Zhao 2018) is improved in our new proposed N-PNLFM waveforms. The bandwidths of the NLFM segment in each N-PNLFM waveform are distributed randomly. In order to suppress the high sidelobes, our proposed N-PNLFM signals consist of a sequence of N-PNLFM subpulses with different subpulse durations. In order to further suppress the repetitive high sidelobes, A particle swarm optimization(PSO) algorithm in (J. J. Liang 2006) is applied to optimize the parameters mentioned above and generate new signals for MIMO radar by reducing the sidelobes in both auto-correlation and cross-correlation functions.

\section{Non-continuous piecewise nonlinear frequency modulation signal model and ambiguity function}

In this section, we first introduce the signal model of our proposed original PNLFM waveform. Then we introduce our proposed N-PNLFM-O-DSD signals for MIMO radar, and the optimization method with a PSO algorithm is also applied.

\subsection{The introduction of original PNLFM waveform}

The time-frequency scheme of original PNLFM waveform is shown in Figure 1. Figure 1(a) and Figure 1(b) show the up and down PNLFM waveform respectively from (Y. Zhao 2018). The $l$ th subpulse of the $m$ th original PNLFM signal can be shown as 
follows:

$$
S_{m, l}(t)= \begin{cases}\mathrm{e}^{j 2 \pi t f_{1, m, l}} \mathrm{e}^{j \pi \mu_{m, l} t^{2}}, & -\frac{T_{\mathrm{p}}}{2} \leq t<-\frac{T_{\mathrm{p}}}{2}+\delta_{\mathrm{T}_{m, l}}, \\ \mathrm{e}^{j 2 \pi t f_{\mathrm{c}}} \mathrm{e}^{j \varphi_{m, l}(t)}, & -\frac{T_{m, l}}{2} \leq t \leq \frac{T_{m, l}}{2}, \\ \mathrm{e}^{j 2 \pi t f_{3, m, l}} \mathrm{e}^{j \pi \mu_{m, l} t^{2}}, & \frac{T_{\mathrm{p}}}{2}-\delta_{\mathrm{T}_{m, l}}<t \leq \frac{T_{\mathrm{p}}}{2} .\end{cases}
$$

where $T_{\mathrm{p}}$ is the duration of each subpulse, $B_{\mathrm{p}}$ is the bandwidth of each subpulse,

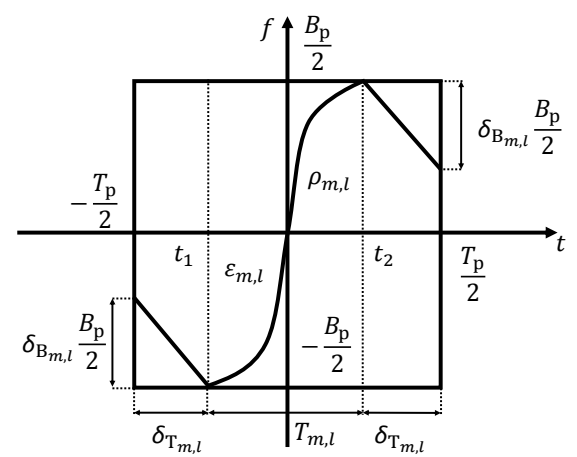

(a)

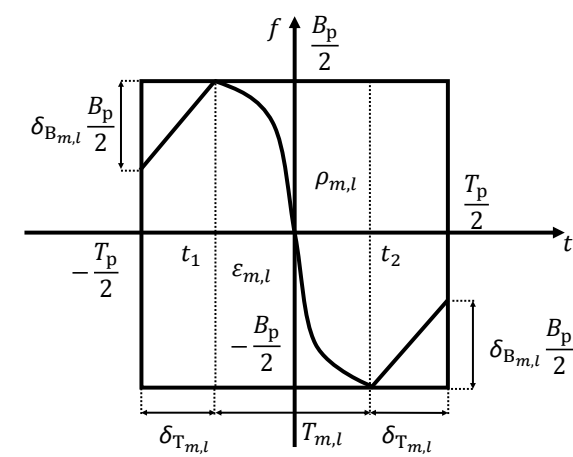

(b)

Figure 1. Time-frequency scheme of PNLFM waveform (a) Original up PNLFM waveform . (b) Original down PNLFM waveform.

$0 \leq m \leq M-1$ and $M$ is the total number of original PNLFM signals, $0 \leq l \leq L-1$ and $L$ is the total sequence number in one original PNLFM signal. The carrier frequencies of the first and third segment are $f_{1, m, l}$ and $f_{3, m, l}$, respectively, and the carrier frequency of the second segment is $f_{\mathrm{c}}, \mu_{m, l}=B_{\mathrm{p}} / T_{\mathrm{p}}$ is the chirp rate. The subpulse bandwidth of the first and third segment is $\delta_{\mathrm{B}_{m, l}}=\delta_{m, l} B_{\mathrm{p}} / 2$. The frequency function of PNLFM subpulse can be expressed as:

$$
f_{m, l}(t)=\frac{\sinh \left(\zeta t / \rho_{m, l}\right)}{\left(1-\epsilon_{m, l}\right) / \epsilon_{m, l} \mathrm{e}^{\left(1 /\left(2 \rho_{m, l}\right)\right)}+\cos \left(\zeta t / \rho_{m, l}\right)} \frac{B_{\mathrm{p}}}{2}
$$

where $t_{1}$ is the beginning point of NLFM segment and $t_{2}$ is the ending point of NLFM segment, and they are shown in Figure (1), $\zeta=20 / T_{\mathrm{p}}$, and $\rho_{m, l}$ and $\epsilon_{m, l}$ are the controllable parameters, $0<\rho_{m, l}<1$ and $0<\epsilon_{m, l}<1$. The phase of the second segment can be expressed as:

$$
\varphi_{m, l}(t)=\frac{\pi \xi_{m, l} \rho_{m, l} B_{\mathrm{p}}}{\zeta} \ln \left(\mathrm{e}^{\frac{1}{\rho_{m, l}}}+\epsilon_{m, l} \cosh \left(\frac{\zeta t}{\rho_{m, l}}\right)-\epsilon_{m, l} \mathrm{e}^{\frac{1}{2 \rho_{m, l}}}\right)
$$

where $\xi_{m, l}$ is the parameter to control the frequency slope of PNLFM subpulse in adjacent time duration, if $l$ is odd, $\xi_{m, l}=1$, and if $l$ is even, $\xi_{m, l}=-1$. The duration of the second segment in the original PNLFM subpulse $T_{m, l}=t_{2}-t_{1}$. It also depends on the frequency function, the duration of the first and third segment in the original PNLFM subpulse can be expressed as $\delta_{\mathrm{T}_{m, l}}=\left(T_{\mathrm{p}}-T_{m, l}\right) / 2$. The chirp rates of the first and third segment are the same and can be defined as $\mu_{m, l}=-\xi_{m, l} \delta_{\mathrm{B}_{m, l}} / \delta_{\mathrm{T}_{m, l}}$. 
And the subpulse frequency of the first and third segments can be shown as follows:

$$
f_{d, m, l}=f_{\mathrm{c}}+(d-2) \xi_{m, l}\left(\frac{\delta_{\mathrm{B}_{m, l}} T_{\mathrm{p}}}{2 \delta_{\mathrm{T}_{m, l}}}+\frac{B_{\mathrm{p}}}{2}-\delta_{\mathrm{B}_{m, l}}\right)
$$

where $d=1,3, f_{\mathrm{c}}$ is the carrier frequency. The time-frequency scheme of the original PNLFM signal is shown in Figure 2(a), the transmitting signal which is composed of original PNLFM subpulses can be defined as follows:

$$
S_{m}(t)=\sum_{l=0}^{L-1} u\left(t-T_{m, l}\right) \times S_{m, l}\left(t-T_{m, l}\right)
$$

where $T_{m, l}$ is the duration of the $l$ th PNLFM subpulse, $u(t)$ represents the rectangle

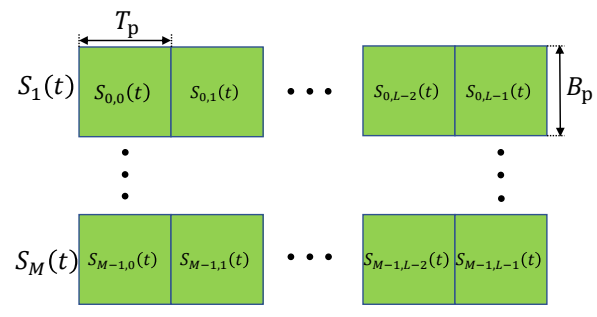

(a)

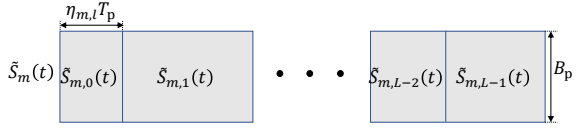

(b)

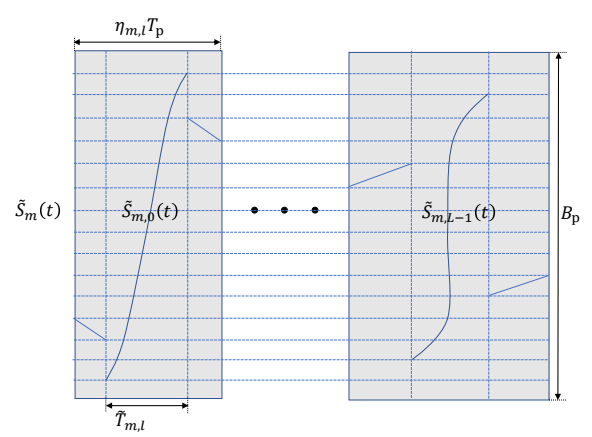

(c)

Figure 2. The time-frequency scheme of PNLFM signals. (a) Original PNLFM signal, $S_{1}(t)$ and $S_{M}(t)$ are the first and $M$ th original PNLFM signals, respectively. (b) Our proposed N-PNLFM-O-DSD signal, $\tilde{S}_{m}(t)$ is the $m$ th N-PNLFM-DSD-O signal with different subpulse duartions. (c) The zoomed in view of our proposed N-PNLFM-DSD-O signal, the LFM segments are orthogonal and the bandwidths of NLFM segments are distributed randomly.

function, $S_{m}(t)$ can be nominated as the original PNLFM signal.

\subsection{Optimization model of our proposed N-PNLFM-O-DSD signal}

The repetitive high sidelobes in the autocorrelation function (ACF) and crosscorrelation function (CCF) are caused by the LFM segments, which are overlaid in the same duration. The smaller the correlations of each segment in the PNLFM subpulses, the lower sidelobes are. Thus, the LFM segment in one sequence of N-PNLFM-O-DSD signal should be orthogonal. The durations of each N-PNLFM-O-DSD subpulse are 
distributed randomly and the time-frequency scheme of N-PNLFM-O-DSD signal can be shown in Figure 2(b). When the duration of each segment is variable, the sidelobes in the ACF and CCF between different segments will be reduced. The zoomed in time-frequency scheme of the N-PNLFM-O-DSD signal is shown in Figure 2(c). It can be seen that the bandwidth of N-PNLFM-DSD-O signal has been separated into $2 L$ sub-bandwidths, therefore, the subpulse bandwidth of each segment is $\tilde{\delta}_{\mathrm{B}_{m, l}}=B_{\mathrm{p}} / 2 L$. In order to keep the diversity of chirp rates, the subpluse time duration of the second segment can be expressed as $\tilde{T}_{m, l}=\eta_{m, l}\left(t_{2}-t_{1}\right)$, where $\eta_{m, l}$ is a controllable parameter to control the each subpulse duration of N-PNLFM-O-DSD signal. In order to further suppress the repetitive high sidelobes, the bandwidths of each N-PNLFM-ODSD subpulse in the second segment are distributed randomly. Thus, the phase of the second segment can be expressed as:

$$
\tilde{\varphi}_{m, l}(t)=\frac{\pi \xi_{m, l} \rho_{m, l} \beta_{m, l} B_{\mathrm{p}}}{\zeta} \ln \left(\mathrm{e}^{\frac{1}{2 \rho_{m, l}}}+\epsilon_{m, l} \cosh \left(\frac{\zeta t}{\rho_{m, l}}\right)-\epsilon_{m, l} \mathrm{e}^{\frac{1}{2 \rho_{m}, l}}\right)
$$

where $\beta_{m, l}$ is a controllable parameter to control the bandwidth of the NLFM segment in the N-PNLFM-O-DSD subpulse. The duration of the first and third segment in the N-PNLFM-O-DSD is $\tilde{\delta}_{\mathrm{T}_{m, l}}=\left(\eta_{m, l} T_{\mathrm{p}}-\tilde{T}_{m, l}\right) / 2$, and the chirp rates of the first and third segment turn to be expressed as $\tilde{\mu}_{m, l}=-\xi_{m, l} \tilde{\delta}_{\mathrm{B}_{m, l}} / \tilde{\delta}_{\mathrm{T}_{m, l}}$. The subpulse frequency of the first and third segments can be shown as follows:

$$
\begin{aligned}
& \tilde{f}_{1, m, l}=f_{\mathrm{c}}-\xi_{m, l}\left(\left(q_{m, l}+1\right) \frac{B_{\mathrm{p}}}{2 L}-\frac{B_{\mathrm{p}}}{2}\right) \\
& \tilde{f}_{3, m, l}=f_{\mathrm{c}}+\xi_{m, l}\left(q_{m, l} \frac{B_{\mathrm{p}}}{2 L}-\frac{B_{\mathrm{p}}}{2}\right)
\end{aligned}
$$

where $q_{m, l} \in\{0,1,2, \cdots, L-1\}$, and when $m$ is fixed, the values of $q_{m, l}$ are different from each other.Then $\tilde{\varphi}_{m, l}(t), \tilde{\mu}_{m, l}$ and $\tilde{f}_{d, m, l}$ will replace the parameters $\varphi_{m, l}(t), \mu_{m, l}$ and $f_{d, m, l}$ in Equation (1), and the expression of our proposed PNLFM-DSD-O signal can be defined as follows:

$$
\begin{aligned}
& \tilde{S}_{m, l}(t)= \begin{cases}\mathrm{e}^{j 2 \pi t \tilde{f}_{1, m, l}} \mathrm{e}^{j \pi \tilde{\mu}_{m, l} t^{2}}, & -\frac{\eta_{m, l} T_{\mathrm{p}}}{2} \leq t<-\frac{\eta_{m, l} T_{\mathrm{p}}}{2}+\tilde{\delta}_{\mathrm{T}_{m, l}}, \\
\mathrm{e}^{j 2 \pi t f_{c}} \mathrm{e}^{j \tilde{\varphi}_{m, l}(t)}, & -\frac{\tilde{T}_{m, l}}{2} \leq t \leq \frac{\tilde{T}_{m, l}}{2}, \\
\mathrm{e}^{j 2 \pi t \tilde{f}_{3, m, l}} \mathrm{e}^{j \pi \tilde{\mu}_{m, l} t^{2}}, & \frac{\eta_{m, l} T_{\mathrm{p}}}{2}-\tilde{\delta}_{\mathrm{T}_{m, l}}<t \leq \frac{\eta_{m, l} T_{\mathrm{p}}}{2} .\end{cases} \\
& \tilde{S}_{m}(t)=\sum_{l=0}^{L-1} u\left(t-\eta_{m, l} T_{m, l}\right) \times \tilde{S}_{m, l}\left(t-\eta_{m, l} T_{m, l}\right)
\end{aligned}
$$


The PSO algorithm is applied to optimize the fitness function with the parameters mentioned above, and the fitness function can be expressed as follows:

$$
\begin{aligned}
& \min _{\boldsymbol{\rho}, \boldsymbol{\epsilon}, \boldsymbol{q}, \boldsymbol{\beta}, \boldsymbol{\eta}}\left\{\sum_{m=0, \tau_{\mathrm{p}} \neq 0}^{M-1} \max \left|R_{S_{m}(t), S_{m}(t)}\left(\tau_{\mathrm{p}}\right)\right|+\sigma_{1} \sum_{m=0}^{m=M-1} \sum_{\tilde{m}=0, \tilde{m} \neq M}^{M-1} \max \left|\chi_{S_{m}(t), S_{\tilde{m}}(t)}\left(\tau_{\mathrm{p}}, f_{\mathrm{d}}\right)\right|\right. \\
& \left.+\sigma_{2} \sum_{m=0}^{m=-1}\left[\sum_{\left\|\tau_{\mathrm{p}}\right\| \neq 0.2 T_{\mathrm{p}}}\left|R_{S_{m}(t), S_{m}(t)}\left(\tau_{\mathrm{p}}\right)\right|^{2} / \sum_{\left\|\tau_{\mathrm{p}}\right\| \leq 0.2 T_{\mathrm{p}}}\left|R_{S_{m}(t), S_{m}(t)}\left(\tau_{\mathrm{p}}\right)\right|^{2}\right]\right\} \\
& \text { s.t. } \quad 0<\epsilon_{m, l}<1<1 \\
& \quad 0.8<\beta_{m, l}<1 \quad 0.2<\eta_{m, l}<1.5
\end{aligned}
$$

where

$$
\begin{aligned}
& \chi_{S_{m}(t), S_{\tilde{m}}(t)}\left(\tau_{\mathrm{p}}, f_{\mathrm{d}}\right)=\int_{-\infty}^{+\infty} S_{m}(t) S_{\tilde{m}}^{*}\left(t-\tau_{\mathrm{p}}\right) \exp \left(j 2 \pi f_{\mathrm{d}} t\right) \mathrm{d} t \\
& R_{S_{m}(t), S_{m}(t)}\left(\tau_{\mathrm{p}}\right)=\int_{-\infty}^{+\infty} S_{m}(t) S_{m}^{*}\left(t-\tau_{\mathrm{p}}\right) \mathrm{d} t
\end{aligned}
$$

where $\tau_{\mathrm{p}}$ denotes the time delay to the target, $f_{\mathrm{d}}$ denotes the Doppler frequency to the velocity of target, ()$^{*}$ represents conjugate, $R_{S_{m}(t), S_{m}(t)}\left(\tau_{\mathrm{p}}\right)$ denotes the ACF, $\chi_{S_{m}(t), S_{\tilde{m}}(t)}\left(\tau_{\mathrm{p}}, f_{\mathrm{d}}\right)$ denotes the CCF, $f_{\mathrm{d}}=0, \sigma_{1}$ and $\sigma_{2}$ are the weighted coefficients to control the proportion of these correlation sidelobes and integral sidelobe ratio (ISLR) in the fitness function mentioned above, respectively. There are five controllable vectors, $\boldsymbol{\rho}=\left\{\rho_{1,1}, \cdots, \rho_{m, l}, \cdots, \rho_{\mathrm{M}, \mathrm{L}}\right\}, \boldsymbol{\epsilon}=\left\{\epsilon_{1,1}, \cdots, \epsilon_{m, l}, \cdots, \epsilon_{\mathrm{M}, \mathrm{L}}\right\}$ $, \boldsymbol{q}=\left\{q_{1,1}, \cdots, q_{m, l}, \cdots, q_{\mathrm{M}, \mathrm{L}}\right\}, \quad \boldsymbol{\beta}=\left\{\beta_{1,1}, \cdots, \beta_{m, l}, \cdots, \beta_{\mathrm{M}, \mathrm{L}}\right\}, \quad \boldsymbol{\eta}=$ $\left\{\eta_{1,1}, \cdots, \eta_{m, l}, \cdots, \eta_{\mathrm{M}, \mathrm{L}}\right\}$. If all the $\eta_{m, l}=1$, it means that the subpulse durations are all the same, it turns to be original PNLFM signal. When $S_{m}(t)=\tilde{S}_{m}(t)$, it is equivalent to N-PNLFM-O-DSD signal. According to the analysis above, the structure of each individual consist of 160 controllable variables, $\boldsymbol{X}=\{\boldsymbol{\rho}, \boldsymbol{\epsilon}, \boldsymbol{q}, \boldsymbol{\beta}, \boldsymbol{\eta}\}$. As the PSO algorithm is applied, the dimension $D=160$. The velocity $V_{i}^{d}$ and position $X_{i}^{d}$ of the $d$ th dimension of the $i$ th particle are updated as follows:

$$
\begin{aligned}
V_{i}^{d}= & w * V_{i}^{d}+c_{1} * \operatorname{rand}_{i}^{d} *\left(\text { pbest }_{i}^{d}-X_{i}^{d}\right) \\
& +c_{2} * \operatorname{rand}_{i}^{d} *\left(\text { zbest }^{d}-X_{i}^{d}\right) \\
X_{i}^{d}= & X_{i}^{d}+V_{i}^{d}
\end{aligned}
$$

where $V_{i}^{d}$ and $X_{i}^{d}$ represent the velocity and position of $i$ th particle, $i=\{0,1, \ldots, I\}$ represents the population number, $d=\{0,1, \ldots, D\}$ represents the dimension, $w$ is the inertia factor, pbest is the best fitness value before the $i$ th particle, zbest is the best fitness value of all the particles. $c_{1}$ and $c_{2}$ are the acceleration constants to control the step towards pbest and zbest position, respectively. $\operatorname{rand} 1_{i}^{d}$ and $\operatorname{rand} 2_{i}^{d}$ are two random numbers ranging form $[0,1]$. All the parameters such as the positions and velocities of original population are initialized. The fitness function has been given in Equation (10), and this is then calculated to determine whether the optimization criteria is satisfied. If the dynamic error of the optimization result in our fitness function close to 0.001, the processing will stop and the controllable vectors mentioned above will be given. The fitness function will be calculated and generate new results until it approaches a fixed value or the processing reaches the end of loop. Finally, the optimization of 
controllable vectors and MIMO radar signals can be achieved, and the high repetitive sidelobes can be suppressed.

\section{Simulation}

In this section, numerical simulations are presented to demonstrate the method of the effectiveness of our proposed N-PNLFM-O-DSD signals, and compare the performance with original PNLFM signals, OFDM PNLFM-DSD signals proposed in Y. Zhao (2018) and distinctive PNLFM signals Y. Zhao (2020). Assuming that $T_{\mathrm{p}}=1 \times 10^{-5} \mathrm{~s}$, $B_{\mathrm{p}}=400 \mathrm{MHz}, F_{\mathrm{s}}=420 \mathrm{MHz}, M=4$, and $L=8$, the total duration of four different signals are $T=8 \times 10^{-5} \mathrm{~s}$. All the time-bandwidth productions of different signals mentioned above are the same. As mentioned above, there are five controllable vectors $\boldsymbol{V}=\{\boldsymbol{\rho}, \boldsymbol{\epsilon}, \boldsymbol{q}, \boldsymbol{\beta}, \boldsymbol{\eta}\}$ in each subpulse. Since there are 32 subpulses in one group of MIMO radar signals, it means that there are totally $D=160$ controllable parameters in the initialization of the PSO algorithm for original PNLFM signals. It is assumed that the population of PSO algorithm is $I=100$ individuals, the acceleration constants $c_{1}=c_{2}=2$, the inertia factor $w=0.2$, and the iteration number is 50 , the original velocities are set in the range $[0,1]$ and the original position are set according to the constraints in Equation (10). In order to suppress the sidelobes, the weighted coefficient is flexible, and here we assume that $\sigma_{1}=3$ and $\sigma_{2}=1$. According to the simulation, when the iteration number is 30 , the fitness function achieves its optimization value.

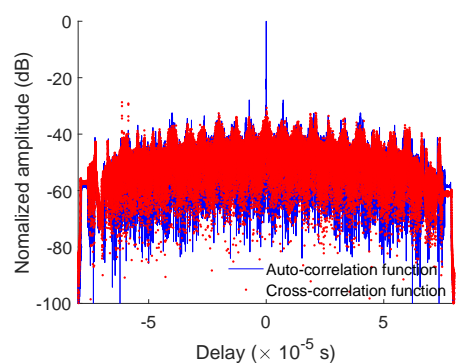

(a)

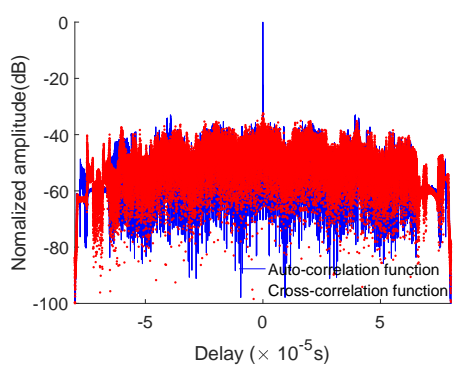

(c)

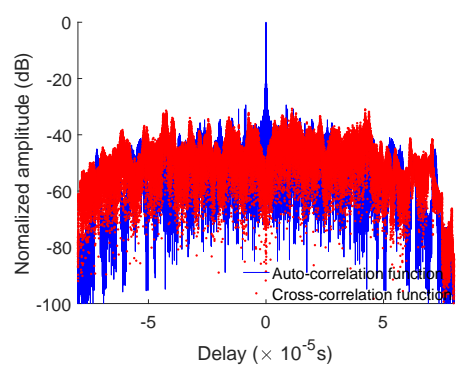

(b)

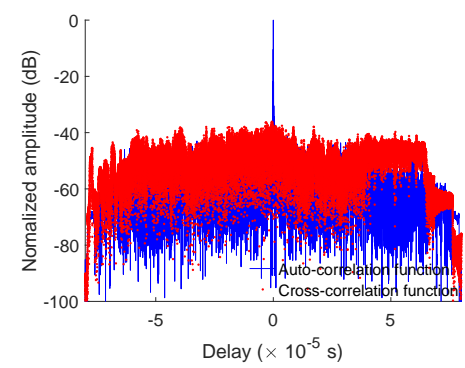

(d)

Figure 3. The ACF and CCF of PNLFM signal with $T_{\mathrm{p}}=1 \times 10^{-5} \mathrm{~s}$. (a) The ACF and CCF of original PNLFM signal. (b) The ACF and CCF of OFDM PNLFM-DSD signal. (c) The ACF and CCF of distinctive PNLFM signal. (d) The ACF and CCF of N-PNLFM-O-DSD signal.

Figure 3(a), 3(b), 3(c) and 3(d) show that all the ACF and CCF of original PNLFM signals, OFDM PNLFM-DSD signals, distinctive PNLFM signals and N-PNLFM-ODSD signal, respectively. It can be observed that there are high repetitive sidelobes 


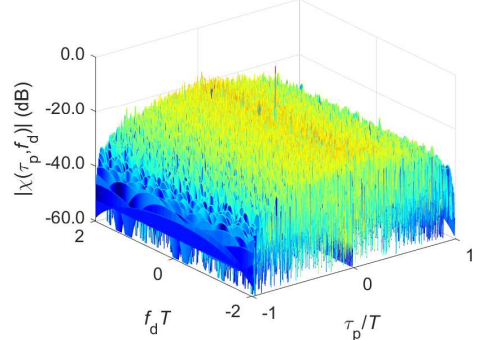

(a)

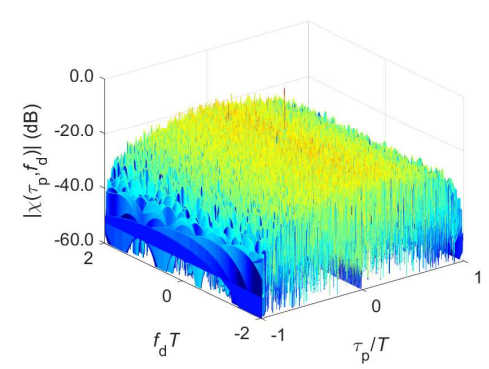

(c)

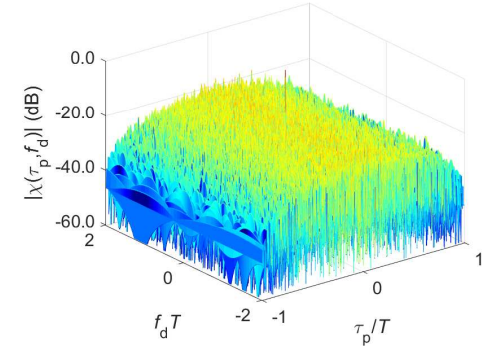

(b)

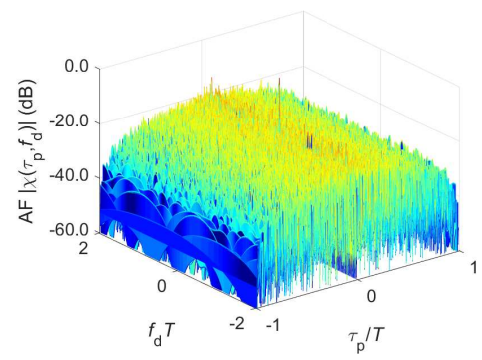

(d)

Figure 4. The AF of PNLFM signal with $T_{\mathrm{p}}=1 \times 10^{-5} \mathrm{~s}$. (a) The AF of original PNLFM signal. (b) The AF of OFDM PNLFM-DSD signal. (c) The AF of distinctive PNLFM signal. (d) The AF of N-PNLFM-O-DSD signal.

in both $\mathrm{ACF}$ and $\mathrm{CCF}$ of original PNLFM signal in Figure 3(a). In our proposed N-PNLFM-O-DSD signals, the duration of each segment are variable and the LFM segments of each subpulse in one signal are orthogonal as shown in Figure 2(c). In addition, the bandwidths of the second segment in N-PNLFM-O-DSD subpulse are distributed randomly as shown in Figure 2(c), too. The high repetitive sidelobes are suppressed significantly as shown in Figure 3(d). Since each PNLFM signal has constant modulus, the peak average power ratio (PAPR) of each normalized PNLFM signal is zero. The OFDM PNLFM-DSD signal has two subpulse in each time domain as shown in Y. Zhao (2018), thus, the PAPR is $3 \mathrm{~dB}$. Meanwhile, the OFDM PNLFMDSD signal still consists of the original PNLFM subpulses, and the original PNLFM waveforms have some overlapped spectrum domains. When the original PNLFM waveforms are regarded as the subpulses, the overlapped spectrum domains can generate autocorrelation sidelobes (ACSLS) or correlation sidelobes (CSLS) with other subpulses. The distinctive PNLFM signal consists of different kinds of PNLFM subpulses, it can increase the domains of freedom in distinctinve PNLFM signals. However, it should be noticed that the PNLFM waveforms proposed in Y. Zhao (2020) also have some overlapped spectrum domains. Although the number of MIMO radar signals are increased, the signals can be further improved. Our new proposed N-PNLFM-O-DSD signal are designed by reducing the overlap of the spectrum in each subpulse, the time-frequency scheme of the N-PNLFM-O-DSD waveform is shown in Figure 2(c). The comparisons of original PNLFM signal, OFDM PNLFM-DSD signal, distinctive PNLFM signal and N-PNLFM-O-DSD signal are shown in Table 1. It can be observed that our proposed N-PNLFM-O-DSD signals achieve better performance and have the lowest ACSLS, CSLS and ISLR.

The AFs of original PNLFM signals, OFDM PNLFM-DSD signals, distinctive PNLFM signals and N-PNLFM-O-DSD signal are also shown in Figure 4, and 
Table 1. Comparisons of original PNLFM signal, OFDM PNLFM-DSD signal, distinctive PNLFM signal and N-PNLFM-O-DSD signal.

\begin{tabular}{|c|c|c|c|c|}
\hline Type of signal & ACSLS (dB) & CSLS (dB) & ISLS (dB) & PAPR $(\mathrm{dB})$ \\
\hline Original PNLFM & -27.89 & -28.7 & -47.13 & 0 \\
\hline OFDM PNLFM-DSD & -29.39 & -32.40 & -47.46 & 3 \\
\hline Distinctive PNLFM & -32.94 & -32.40 & -48.25 & 0 \\
\hline N-PNLFM-O-DSD & -41.33 & -37.10 & -48.53 & 0 \\
\hline
\end{tabular}

$\left|\chi\left(\tau_{\mathrm{p}}, f_{\mathrm{d}}\right)\right|$ is the amplitude of AFs. It can be observed that there are high sidelobes in the direction of Doppler delay as shown in Figure 4(a), the signal is composed of original PNLFM signals. By applying our new time-frequency scheme with our proposed N-PNLFM-O-DSD signals, the interference sidelobes which are caused by each linear segment and nonlinear segment are reduced as shown in Figure 4(d). Thus, the sidelobes in ACF, CCF and AF of N-PNLFM-O-DSD signal are reduced simultaneously. Comparing with the OFDM PNFLM-DSD signals and distinctive PNLFM signals mentioned above, all the AFs of these three signals resemble a thumbtack. OFDM PNLFM-DSD signals can be applied into MIMO radar when all the signals are orthogonal, however, perfect orthogonal signals are hard to achieve, and it's an ideal situation in simulation. Distinctive PNLFM signals can increase different kinds of signals which achieve lower ACSLS and CSLS, while it takes the cost of using the overlapped time-frequency scheme subpulses. If MIMO radar need large number of signals with low ACSLS and CSLS, it's a good choice to use distinctive PNLFM signals. To our new proposed N-PNLFM-O-DSD signals, it only consists of one kind of signals. However, the new subpulses have the lowest overlapped time-frequency scheme, and N-PNLFM-O-DSD signals achieve the lowest ACSLS and CSLS. When it comes to a small scale of radar system with a few of MIMO radar signals, it's better to use our new proposed N-PNLFM-O-DSD signals.

\section{Conclusion}

Reducing sidelobes in MIMO radar systems through waveform design is an important aspect of creating an overall more capable radar sensor. In this paper, one N-PNLFMO-DSD waveform has been proposed for using in MIMO radar. Each subpulse of NPNLFM-O-DSD signals have different durations, making the chirps rates of each LFM segment different. In addition, the LFM segments of each subpulse in one N-PNLFMO-DSD signal are orthogonal in the time-frequency scheme and the bandwidths of the second segment in each subpules are distributed randomly. Simulation results have been presented to illustrate that the high sidelobes in original PNLFM signals have been suppressed through the applied PSO algorithm. Our newly proposed waveforms have lower ACSLS, CSLS and ISLR. Overall this means that these waveforms would enable a MIMO radar system to perform better in potential applications such as SAR imaging. The impact of these waveforms on performance in these different cases will be the focus of future research.

\section{Funding}

This work was supported by the National Natural Science Foundation of China under Grant 61671246; under Grant 61801221 and Natural Science Foundation of Jiangsu Province of China under Grant BK20170855. 


\section{References}

Boukeffa, S., Y. Jiang and T. Jiang, 2011. "Sidelobe reduction with nonlinear frequency modulated waveforms," 2011 IEEE 7th International Colloquium on Signal Processing and its Applications, 399-403.

Chen, C., and P. P. Vaidyanathan, 2008. "MIMO Radar Ambiguity Properties and Optimization Using Frequency-Hopping Waveforms," IEEE Transactions on Signal Processing, 56 (12), 5926-5936.

Cui, G., H. Li and M. Rangaswamy. 2014. "MIMO Radar Waveform Design With Constant Modulus and Similarity Constraints," IEEE Transactions on Signal Processing, 62 (2) 343353.

De Witte, E. and H. D. Griffiths, 2004. "Improved ultra-low range sidelobe pulse compression waveform design," Electronics Letters, 40 (22) 1448-1450.

Gao, C., K. C. Teh and A. Liu, 2016. "Double-Modulated Frequency Modulation Waveforms for MIMO Radar," IEEE Geoscience and Remote Sensing Letters, 13 (12) 2024-2028.

Gao, C., K. C. Teh, A. Liu and H. Sun, 2016. "Piecewise LFM waveform for MIMO radar," IEEE Transactions on Aerospace and Electronic Systems, 52 (2) 590-602.

Gao, C., K. C. Teh and A. Liu, 2017. "Piecewise Nonlinear Frequency Modulation Waveform for MIMO Radar," IEEE Journal of Selected Topics in Signal Processing, 11 (2) 379-390.

Griffiths, H. D. and L. Vinagre, 1994. "Design of low-sidelobe pulse compression waveforms," Electronics Letters, 30 (12) 1004-1005.

Kim, J., M. Younis, A. Moreira and W. Wiesbeck, 2013. "A Novel OFDM Chirp Waveform Scheme for Use of Multiple Transmitters in SAR," IEEE Geoscience and Remote Sensing Letters, 10 (3) 568-572.

Kim, J., M. Younis, A. Moreira and W. Wiesbeck. 2015. "Spaceborne MIMO Synthetic Aperture Radar for Multimodal Operation," IEEE Transactions on Geoscience and Remote Sensing, 53 (5) 2453-2466.

Krieger, G.. 2014. "MIMO-SAR: Opportunities and Pitfalls," IEEE Transactions on Geoscience and Remote Sensing, 52 (5) 2628-2645.

Li, H., Y. Zhao, Z. Cheng and D. Feng, 2017. "OFDM Chirp Waveform Diversity Design With Correlation Interference Suppression for MIMO Radar," IEEE Geoscience and Remote Sensing Letters, 14 (7) 1032-1036.

Li, J., and P. Stoica. 2007. "MIMO Radar with Colocated Antennas," IEEE Signal Processing Magazine, 24 (5), 106-114.

Liang J. J., A. K. Qin, P. N. Suganthan and S. Baskar. 2006 "Comprehensive Learning Particle Swarm Optimizer for Global Optimization of Multimodal Functions," IEEE Transactions on Evolutionary Computation, 10 (3), 281-295.

Sun, S. and A. P. Petropulu. 2015. "Waveform Design for MIMO Radars With Matrix Completion," IEEE Journal of Selected Topics in Signal Processing, 9 (8) 1400-1414.

Wang, J., L. Chen, X. Liang, C. Ding and K. Li, 2015. "Implementation of the OFDM Chirp Waveform on MIMO SAR Systems," IEEE Transactions on Geoscience and Remote Sensing, 53 (9) 5218-5228.

Wang, W., 2015 "MIMO SAR OFDM Chirp Waveform Diversity Design With Random Matrix Modulation," IEEE Transactions on Geoscience and Remote Sensing, 53 (3) 1615-1625.

Zhao, Y., X. Lu, J. Yang, W. Su and H. Gu. 2018. "OFDM waveforms designed with piecewise nonlinear frequency modulation pulse for MIMO radar" International Journal of Remote Sensing, 39 (23) 8746-8765.

Zhao, Y., X. Lu, M. Ritchie, W. Su and H. Gu. 2020. "Suppression of sidelobes in MIMO radar with distinctive piecewise non-linear frequency modulation sub-carrier" International Journal of Remote Sensing, 41 (1) 353-372. 